\title{
Comparative Study of the Student's Design Process: Implications for the Teaching of the Later Phases of the Mechanical Engineering Design Process
}

\author{
Damien Motte \\ Division of Machine Design, \\ Lund Institute of Technology \\ at Lund University \\ damien.motte@mkon.lth.se
}

\author{
Per-Erik Andersson \\ Division of Machine Design, \\ Lund Institute of Technology \\ at Lund University \\ per-erik.andersson@mkon.lth.se
}

\author{
Robert Bjärnemo \\ Division of Machine Design, \\ Lund Institute of Technology \\ at Lund University \\ robert.bjarnemo@mkon.lth.se
}

\begin{abstract}
Most methods that guide the designer through the later phases of the design process are general in nature, and it is up to the designer to organize the design work using the tools and techniques available. This process also relies greatly on experience, which is quite a challenge for students, who are mostly novices in the area. In a comparative study, the evolution of the experience and skills acquired by the students in performing design tasks during the embodiment design and detail design phases has been analyzed. The results indicate the main directions for improvement in teaching the later phases of the mechanical engineering design process.
\end{abstract}

\section{Introduction}

The mechanical engineering design process that is, the sequences of the activities required to design an artifact from its specifications to a product ready to be manufactured - that is taught to the student is often a decisive moment in his or her education. Of course, the mechanical engineering designer may use and learn several individual new techniques during a life-long carrier, but the way of organizing and sequencing his or her activities will tend to change more slowly and more difficultly, and is thus greatly determined by what has been learned at the university. The mechanical engineering design process, or design process for short, is deeply linked with the designer's experience, and is therefore time-consuming to assimilate and hard to change. Close attention must be paid to what the student needs to know, as well as to what he is ready to understand and assimilate and on which points the teacher must be resolute.

Little is actually known about how the student reasons and absorbs the concepts taught in class. The feedback provided by examinations helps to review the weaknesses of the students and consequently im- prove the course, but this is actually limited by the extent of the assignment task, which, in addition, only delivers the design process result and gives few insights into how the design task has been carried out. Atman et al. [1] studied the behavior of students more deeply by carrying out isolated experiments. Their successive studies on students now provide an initial mapping of the evolution of the students' capacity for designing from the stage of freshmen to that of seniors (last-year or graduate student). Nevertheless, these studies focus on the conceptual phase of the design process. This phase is crucial because the students need to acquire the skills of questioning the task assignment, searching for information, generating several alternatives and frequently iterating between the different steps of design (see also Adams et al. [2]). But the tasks that characterize the later phases of the design process (the embodiment design and detail design phases) are different. The designer has to gather the whole body of knowledge acquired in different disciplines (solid mechanics, materials, applied mathematics...) in order to embody and dimension the product-to-be. The designer also needs to be familiar with a whole set of techniques specific to each subproblem (see e.g. Pahl \& Beitz [3]). This synthesis activity demands a different way of thinking.

This paper presents an observation of the evolutionary pattern of the students' ability to design by comparing juniors and seniors, based on an explorative study of the students' design process. This paper is partly based on a previous study reported in [4] and [5], which differentiated between the design activities performed by students and experts but did not differentiate between students.

Four dimensions of the design activity are investigated. The first one is the observation of the design activity viewed as a problem-solving process. The second and third are the design strategies and tactics that the students develop, or apply through the resolution of a design task. Finally, the techniques used by 
the designers are listed, considering the following categories: basic rules (clarity, simplicity and safety), principles or guidelines, and factors concerning the product life cycle (e.g. production, transport, recycling).

The implications of the findings for the teaching of the design process during the embodiment design and detail design phases are then discussed.

\section{Related Work}

A whole body of works focuses on, and reflects over, the difficulties of teaching and learning the design activity, taking into account the advancements in the fields of cognitive sciences, sociology and education $([2,6-8])$.

Concerning the problem-solving process, most of the research works in that area focus on the conceptual design phase. [9] showed that the more time students spent on problem scoping, the better the result. [10] showed that the design process could be described as an incremental process: the students understand the problem progressively and refine the alternatives (further developed in [11]). [1], further comparing freshmen and seniors, demonstrated that the students' design skills had improved (considering design outcome) with time. The design process was characterized by more information gathered, more alternatives developed, more iterations and more time dedicated to evaluation and decision. [12] studied the correlation between the quantity of sketches and design outcome by seniors. Casakin \& Goldschmidt in [13] are working on the use of visual analogy as a problemsolving strategy. In a review published in [14], the main shortcomings observed for both students and experts reported were: early appearance and persistence of a kernel idea; design fixation (inclination to stick with early satisficing solutions); lack of flexibility in the designer's thinking behavior; superficial assessment, and subjective judgment.

In [4], a model was developed that describes the problem-solving pattern of designers (students and experts) during the embodiment design and detail design phases. 1) Contrary to the conceptual design phase, the problem scoping is very rapid, and most of the designers do not question the stated problem or come back to it during the design activity. 2) Very few alternatives are developed; the development of a solution is an interplay between the synthesis of the solution and its mechanical modeling. 3) Evaluation is made along the solution generation activity (and not at the end of the activity) by implicit or explicit criteria. 4) The detail drawing activity actually plays the role of control of the solution: everybody had to come back to the solution generation activity. This seems to explain why Yang [12] found that the presence of dimensioned sketches early in the conceptual design phase led to better design outcome. Detail drawing is the first moment where the designer needs to consider all dimensions, proportions, and interfaces.

There have been few observations of the designer's strategies and tactics during the embodiment design and detail design phases. Motte et al. [5] review the main findings on this design research area. Prescriptive strategies remain at a general level (e.g. $[3,15])$ or highlight the difficulty of developing specific strategies and tactics, especially at the early embodiment design phase $([16,17])$. [5] reports that a common design strategy could be induced from the designers studied. A set of tactics has been extracted from observing the designers. Also weaknesses have been listed. Strategy, tactics and weaknesses are summarized in Table 1.

\section{The teaching of the embodiment design and detail design processes}

At Lund University, Sweden, students wishing to become mechanical engineering designers follow two years of general lectures (applied mathematics, physics, solid mechanics...) before specialization. In the third year, they learn about product planning and conceptual design in product development, as presented in [19]. Although the students learn tools and techniques useful for the embodiment design and detail design activities from the very first year, they really tackle the later phases of the design process during the last year, with the lectures on product architecture and form giving.

At this stage, the overall principle that is behind the teaching is the principle of "learning by doing" together with design cases study. Besides the general strategies mentioned in the literature $([3,15])$, there is not really any developed method that supports the designers for the embodiment design and detail design phases. The students learn a series of basic rules, guidelines and principles that help, but do not guide him or her through the embodiment and detailing of a technical system. Thus this is a study of different designs, training in different design tasks and projects (a six-month project or the M.Sc. thesis) that give the student the experience and sensitivity needed for designing.

The basic rules the designers need to have in mind during the design activity are simplicity, clarity and safety $([3,17,20])$. Briefly, Simplicity means that the design must be simple to analyze and understand, with few components; clarity means that the behavior of the technical system must be easy to predict; safety means that the designer must take into account 
Table 1. Strategies, tactics and weaknesses (excerpt from [5]).

\begin{tabular}{|c|c|c|}
\hline Strategies & Tactics & Weaknesses \\
\hline $\begin{array}{l}\text { General Strategy: } \\
\text { Rapid understanding of the problem. } \\
\text { Considering, very early in the proc- } \\
\text { ess, the shapes of the parts and their } \\
\text { interactions. } \\
\text { Concrete choice of materials. } \\
\text { Optimized choice of standard compo- } \\
\text { nents. } \\
\text { Dimensioning of the joints. } \\
\text { Variations: } \\
\text { Dimensioning by experience or by } \\
\text { mechanical analysis. } \\
\text { Often depth-first strategy. } \\
\text { Clear method that is loosely followed. }\end{array}$ & $\begin{array}{l}\text { Think in terms of standard compo- } \\
\text { nents. } \\
\text { Thinks in terms of concrete shapes. } \\
\text { Document the work. } \\
\text { Detail drawing. } \\
\text { Use of basic rules. } \\
\text { Criteria: } \\
\text { Minimization of costs, } \\
\text { Avoid unique part. } \\
\text { Take into account production. } \\
\text { Wait until late before using principles } \\
\text { and guidelines. }\end{array}$ & $\begin{array}{l}\text { Do not ask beyond the assignment. } \\
\text { Do not plan design activity (at an opera- } \\
\text { tional level). } \\
\text { Do not use a developed objective func- } \\
\text { tion. } \\
\text { Check activity considered as secondary. } \\
\text { Basic rules often followed only at the } \\
\text { beginning of the design process. } \\
\text { No check for other factors than "costs" } \\
\text { and "manufacturing/assembly". } \\
\text { Students: } \\
\text { Seldom check their design } \\
\text { Design knowledge not mastered (lack of } \\
\text { experience) }\end{array}$ \\
\hline
\end{tabular}

component reliability, function reliability, operational safety, and environmental safety [3].

The guidelines concern specific areas generally developed under the denomination of "design for X". Standard guidelines that help the designer dimensioning joints, like VDI 2230 [21], estimating costs, like VDI 2225 [22], or analyzing the quality and reliability of the product ([23-25]), can be included as well. Guidelines concern ultimately "ways of dealing with some physical and natural effects/phenomena like corrosion, wear and thermal expansions" [14].

Finally, the principles are practices, or rules of thumb, that have been proved very useful to an effective design (e.g. [26-28]). The first principle students usually learn is the following: "if a force or moment is to be transmitted from one location to another, with the minimum possible deformation, then the shortest and most direct force transmission path is the best" $([26,3])$.

This illustrates the extent to which the design process of the embodiment design and detail design phases is a patchwork of elements that the student learns. How and when to use these basic rules, guidelines and principles is left up to the student. By comparing juniors (students that are learning form-giving and product architecture) and seniors (students who have completed the course and have almost "one year experience"), this paper gives insights into the progress made by the students. This comparison, made through the four dimensions mentioned earlier problem-solving process, design strategies, tactics, and techniques used, and coupled to the comparison between students and experts developed in [4] and [5] can serve as a basis for improving the teaching of the process of the embodiment design and detail design phases.
The next section presents the methods used to observe the evolution of the students' design skills.

\section{Methods}

Two methods were employed. This paper is principally based on the first: the analysis of experiments where designers were asked to solve a design task. On the fringe of these experiments, sketches of a design task carried out by juniors attending the lecture on form giving have been analyzed.

\subsection{Controlled experiments}

Studying the designers under controlled experiments makes it possible to focus on the design process and monitor factors that could bias the analysis. Six experiments have been carried out, with three experts and three students (two seniors and one junior). The junior was about to begin the course on form giving, while the two seniors were completing their M.Sc. theses. The designers had to embody and dimension a support for a hydraulic cylinder. The support had to stand beside the installation (see Figure 1). The full experiment protocol is described in [29].

The designers were videotaped and were asked to "think aloud", that is, to describe what they were doing. What they said was then transcribed and analyzed by the verbal protocol analysis method [30]. [31] demonstrated verbal protocol analysis as a relevant method for studying students' design activity. Verbal protocol analysis consists in the segmentation of the verbal protocol into episodes that represent a single action. The episodes are then analyzed with the help of a set of categories, or coding schemes, each representing a basic action. The repetition and the sequences of the basic actions are then interpreted. 
A coding scheme has been developed for the study of the design activity modeled as a problemsolving activity [4], and another coding scheme has been developed for the study of the designers' strategies and tactics [5].

Each time a designer applied a basic rule, guideline or principle, the nature of this basic rule, guideline or principle and the moment it was applied were reported. So it was with the factors the designers took into account during the design process. These factors were adapted to the design task of the experiment from those given in [3] (p. 206), among others: manufacturing/assembly, transport, operation, recycling, costs, schedules.
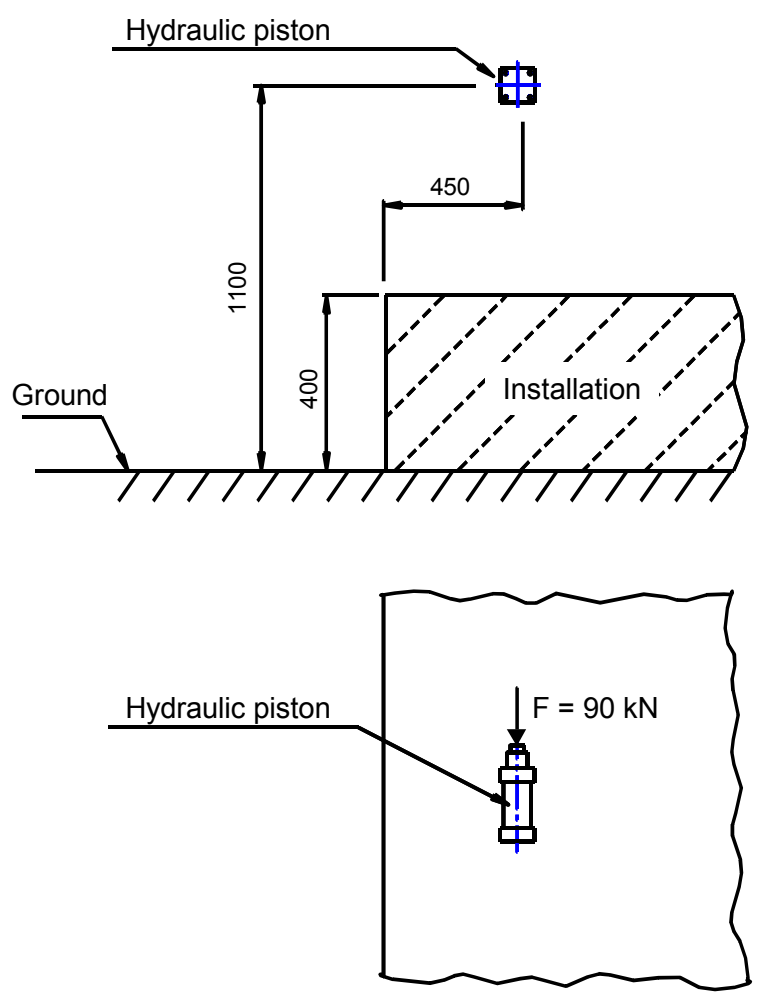

Figure 1. Sketch of the problem delivered with the assignment [29].

\subsection{Design task sketches}

The same design task as the one used for the experiments was given to the students as an obligatory examination task. They had three weeks to submit their solution (a fully dimensioned support for the hydraulic cylinder) and the theoretical time they had at their disposal to work on the problem, given that the volume this course represented in the study curriculum was equivalent to 3-4 days of fulltime work. The day the design task was distributed and discussed in class, the students were asked to sketch the first idea they had for a solution. The drawing of the final solution delivered after three weeks could then be compared to the first sketch. This sketch could even be compared to the first sketches of the designers who participated in the experiments.

\section{Results and discussion}

The results are presented following the four dimensions mentioned above: problem-solving activity, design strategies, tactics, and techniques used (basic rules, guidelines, principles and other factors). The results from the comparison of the design sketches are presented later.

\subsection{Problem-solving activity}

Once the verbal protocols are coded, that is once an action or category has been attributed to every episode, the sequence of problem-solving actions can be visualized. Figure 2 shows the sequence of actions performed by the junior; Figure 3 shows the sequence of actions performed by a senior. The interruptions that can be observed on the charts correspond mainly to social behavior episodes, when the designer loses attention, justifies his faults or simply relaxes [29]. The episodes that could not be coded (because of bad recording for example) represent a negligible part of the total number of episodes. In this section, the overall strategy developed by the designer is discussed first, and then the individual episodes.

If on the whole the junior has the same strategy (see section 2) as the other designers, his progress was very loosely structured. The student went thoroughly through the task assignment, but rapidly lost focus on the expected results of the assignment (among other, a detailed sketch of a solution), before coming back to it. The seniors, to that extent, acted like the experts. Nevertheless, the interplay between synthesis and mechanical modeling, followed by dimensioning, which is described in [4], was present in the problemsolving activity of the junior. This seems to indicate, as the student had almost no experience, that this pattern of actions is acquired "naturally" or prior to design lectures, that is, in any case, not acquired by experience.

The junior was the only designer who came back to problem understanding (categories Irp, Es, Sp in Figure 2) late in the design process. The student made a thorough rereading of the problem statement, checking if his design process and solution corresponded to the requirements. However, the junior developed what Bender \& Blessing [32] called adhocism. The junior tried rather to adapt the problem to his solution and his skills (see also [4]). The problem understanding episodes that can be seen after the first half hour often 


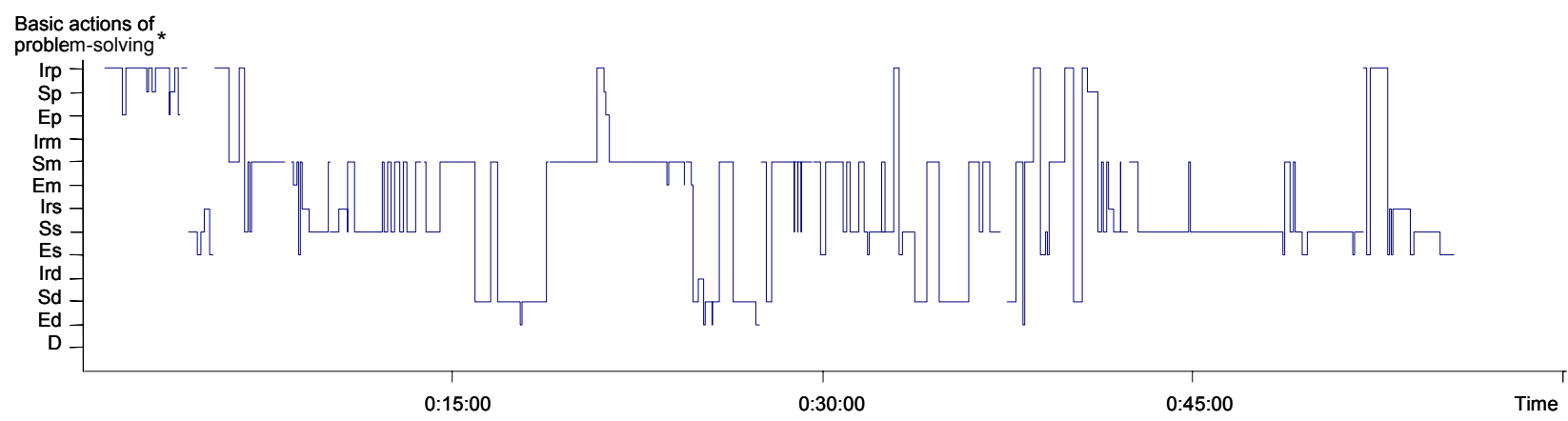

Figure 2. Problem-solving activity of the junior.

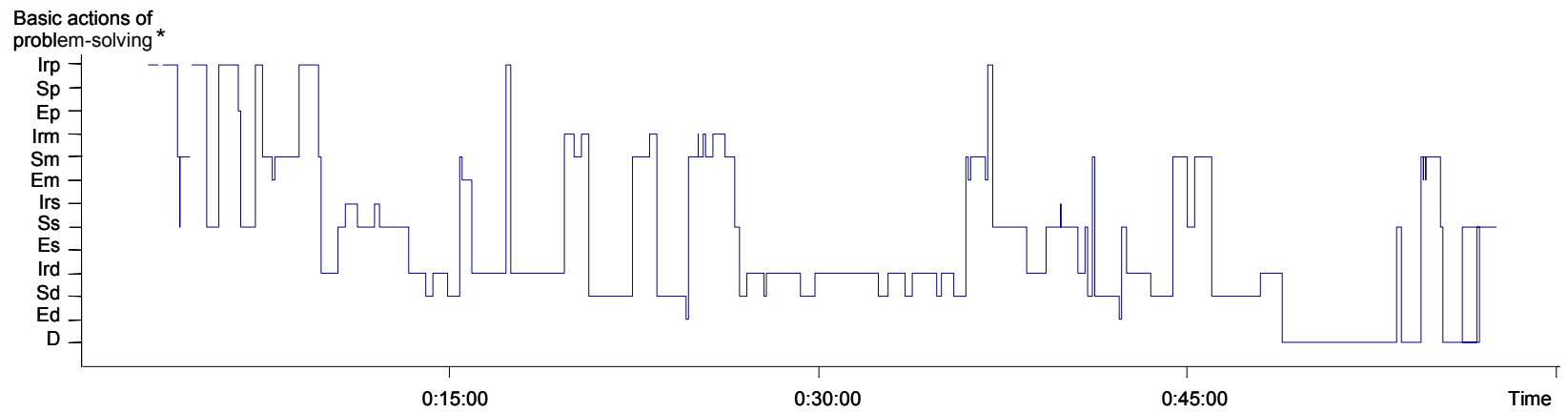

Figure 3. Problem-solving activity of a senior.

* Sp: problem reframing; Sm: mechanical modeling of the solution; Ss: Synthesis of the solution; Sd: dimensioning; D: detail drawing; Irp, Irm, Irs, Ird: information search episodes concerning Sp, Sm, Ss, Sd respectively; Ep, Em, Es, Ed: evaluations/decisions concerning Sp, Sm, Ss, Sd respectively (see [4]).

became questions that were preliminaries to a kind of negotiation with the experimenter. That the junior did not go into detail drawing is an illustration of this behavior; the interpretation of the problem statement led to the fact that his drawings - and then solution dimensioning — did not need further refinement (see Figure 6). The seniors did not behave this way. They sometimes admitted the difficulty of the exercise but never tried to change the assignment.

The information search episodes (Irp, Irm, Irs, Ird, see Figure 2) of the junior were fewer in number and time than the seniors, but this was largely due to the fact that the junior did not try to dimension everything. Thus he did not have to search for profiles, materials, etc. Most of the information search episodes were dedicated to problem understanding.

In comparison with the seniors, the junior spent much more time on mechanical modeling $(\mathrm{Sm})(30 \%$ of the time against $12 \%$ for the senior, whose problem-solving activity is represented in Figure 3). This was clearly due to a lack of knowledge and experience. The seniors spent more time on $\mathrm{Sm}$ than the experts, but that was rather due to the fact that the seniors' solutions were more complicated [29].
There were more evaluation moments (Ep, Em, Es, Ed), both in time and number, than for the seniors. But this cannot at present be interpreted as a characteristic evolution between juniors and seniors; more experiments are needed in this area. The evaluation episodes of the juniors are indeed similar to the evaluation episodes of the experts; but due to the radically different sequences of basic actions that they had, it would be harsh to try to find a correlation between the evaluation episodes and the evolution of the designers' behaviors.

\subsection{Design strategies and tactics}

The second and third dimensions studied were the design strategies and tactics applied by the designers. This was done with the help of a new coding scheme. Figure 4 represents the sequence of the basic design tasks performed by the junior; Figure 5 represents the sequence of the basic design tasks performed by a senior.

The strategy deployed by the experts is presented in Table 1. One senior adopted the same pattern, while the other one, who did not dimension his solution, skipped several points (optimized choice of standard 


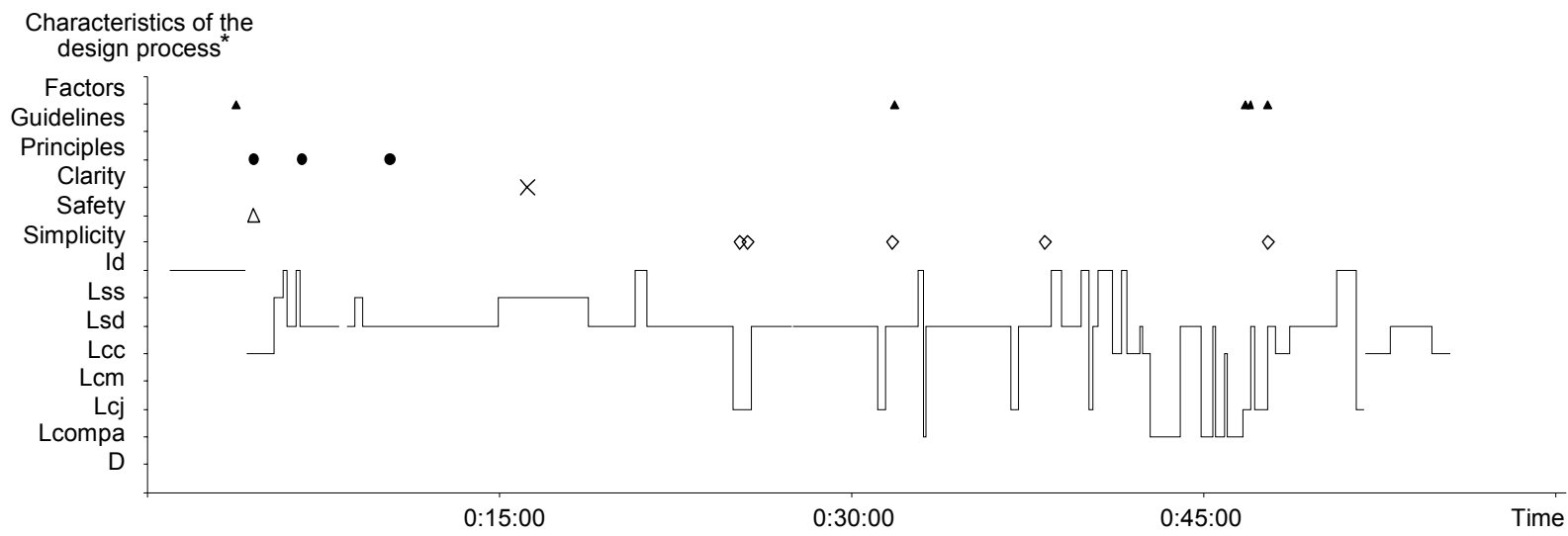

Figure 4. Basic design tasks performed by the junior.

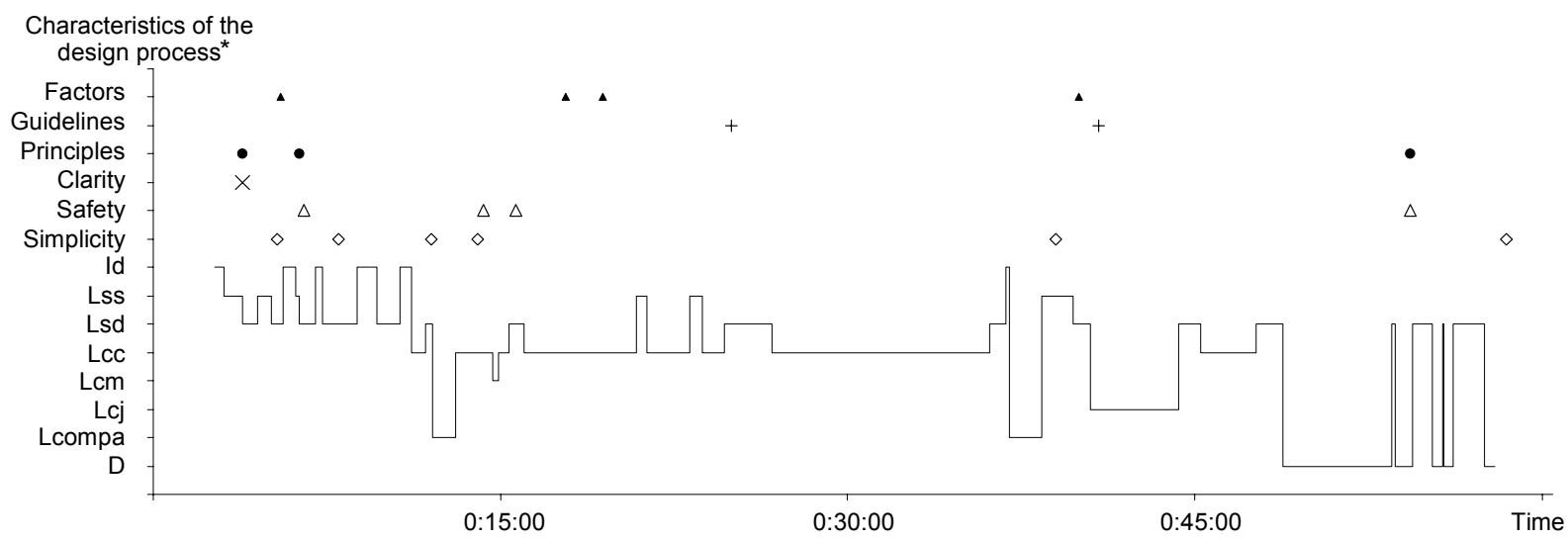

Figure 5. Basic design tasks performed by a senior.

* Id: problem identification; Lss: scale of spatial constraints; Lsd: synthesis; Lcc: choice of components; Lcm: choice of material; Lcj: choice of joints; Lcompa: ensure compatibility/interface; D: detail drawing (see [5]).

components, for example). The junior did not try very hard to structure his process. The two seniors began by embodying the support, as did the experts, although more loosely: they did not attach importance to the interfaces between the parts and the environment. The junior began with the choice of the mountings of the hydraulic cylinder (Lcc episodes at the beginning of the design process, see Figure 4), before beginning the embodiment.

The junior did not try to choose standard components. The beams of the support are supposed to be larger at their base (Figure 6). This leads not only to manufacturing difficulty, but the dimensioning itself becomes very complex. The necessity of simple and clear designs (which are by the way a consequence of the simplicity and clarity rules, see below) that make calculations easier has been integrated by the senior, who chose standard I-beams. Still, the seniors seem to have neglected the interfaces problem. If we compare the design made by a senior (Figure 7) and the design made by an expert (Figure 8), the interface problems are treated from the beginning by the expert, while they are neglected by the seniors who, like the junior, develop specific parts.

It is worth mentioning that by the end of the experiment the junior proposed the use of I-beams, but still maintained his requirements (larger at the base). $\mathrm{He}$ actually postponed the problem, assuming that someone else would take care of it (maybe the manufacturing department). An interesting question arises concerning the perception of the concept of (mechanical engineering) design by the students. In this case, the creative view of design seemed to be emphasized to the detriment of a more concrete perspective: a design solution must work. The seniors, however, take this into account: the problems they neglected were due to lack of experience (the interface problems, for example), not to conscious postponement of the problem. This is probably partly due to design cases studied in class (design cases show how every detail is 


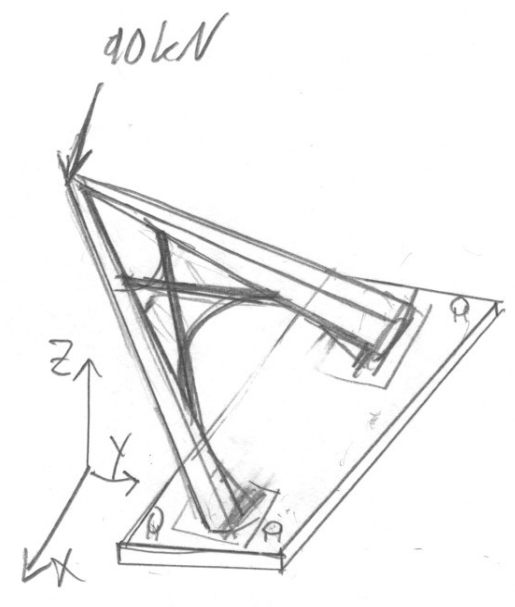

Figure 6. Junior's embodiment of the support.
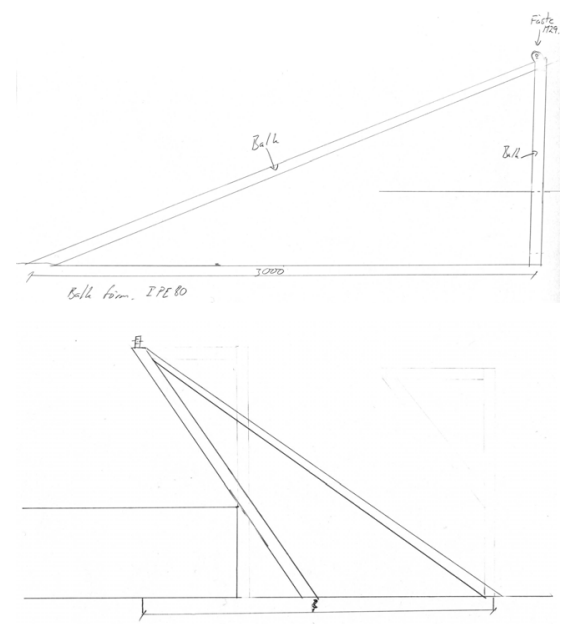

Figure 7. Embodiment of the support of one senior.

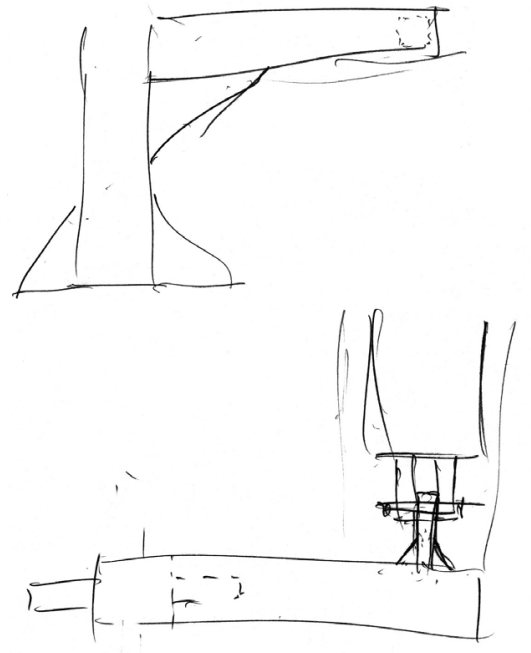

Figure 8. Embodiment of the support of one expert. [29] important), but also to the 6-month the product development project and M.Sc. thesis that follow, and which are done in collaboration with industry.

Surprisingly, the junior did not document his work very much. It was assumed that two years of physics and mathematics would have given some rigor in the task performed, but this was not the case. This may be due to the fact that, the design process being loose, the junior did not know what was important to write down and what was not. The seniors had the same behavior. This resulted in loss of time for both.

Finally, the seniors (and the experts) always had in mind one function to optimize while designing, namely the costs. The experts had a more sophisticated model, taking into account manufacturing and assembly.

\subsection{Basic rules, guidelines, principles and other factors}

The junior was led by the simplicity rule as many times as the senior (around 5 times in one hour). However, this rule was used mostly when the designer had trouble with his design or when dealing with details. This was sometimes the case for the senior, but the rule was used for decisive elements of the system. Like the experts, the seniors let the simplicity rule constrain their design. This rule seems to be quickly assimilated by the students.

The rule of clarity is the least understood. The junior used it once, but so did the senior. Clarity is a difficult concept that is taught through design cases. There may be a need to teach it in a different manner so that the student assimilates it more rapidly.

The safety rule was used only once, against three times for the seniors. The experts, on the other hand, used this rule frequently. There seems to be a need to insist on the dimensions of the safety rule (component reliability, function reliability, operational safety, and environmental safety).

Seniors and experts overall used the basic rules at the beginning and at the end of the design experiment. They have in mind at the beginning the necessity of simplicity, clarity and safety, but forget about them as the design progresses. They tend to stick to parts of the solution that they try to dimension, rather than simplifying or changing. At the end of the experiment, the designers did a detail drawing of their solution and then, when hidden faults became visible, they had to come back to synthesis and use the basic rules again [5]. The junior shows a rather continuous use of the basic rules. This is due to the fact that, as mentioned earlier, his design process was more loosely structured: the junior sometimes began to be interested in a new part of the solution, and then once again began the synthesis (and mechanic modeling activity). 
The junior did not apply any guideline. One senior applied 2 guidelines (one for buckling analysis, one for welding). This tends to show that the students understand the importance of the guidelines. It is worth noticing that the seniors would have used FEM, if these tools had been available, instead of estimate calculations.

The students used the principles of direct force and stability [3]. The design task of the experiments was not adapted to the use of many different principles. A new experiment with a different design task needs to be set up in order to observe how well the students assimilate the principles they learn.

The junior did consider more factors than the seniors: production/assembly, costs, maintenance and dismantling, while the seniors only considered production/assembly and costs. However, the junior considered these factors for details, while the seniors used them as clues for designing. Many other factors (see section 4.1) important for the quality of the design outcome were nevertheless neglected. This holds as well for the experts. The checklist presented by Pahl \& Beitz [3] needs to be more present during the teaching of the later phases of the design process.

\subsection{Design Sketches}

Parallel to the experiments, other students attending the lecture on form giving were asked to draw a sketch of their first idea. This was compared to their final design. Figure 9 is the first sketch of one student. Figure 10 is the final solution he delivered for the examination. As can be seen, the design is far simpler (fewer parts, standard components) and clearer (the support is easier to analyze). The result was similar for most of the other students. This is without doubt largely due to the analysis and calculation problems the students would have had with their first design, illustrating the strength of the "learning by doing" method.

However, many designs remained incomplete: there is no fixation for the hydraulic cylinder; there is no fixation on the ground. The welding of the I-beam on the rounded corner of the VKR-beam (see Figure 10) can be problematic. This confirms our previous remarks on the necessity to emphasize the interface problem.

\section{Conclusion}

In this paper the evolution of the design skills and experience of students during the later phases of the design process has been analyzed. The design processes of a junior and two seniors were compared along four dimensions: problem-solving process, design strategies, design tactics and application of basic rules,
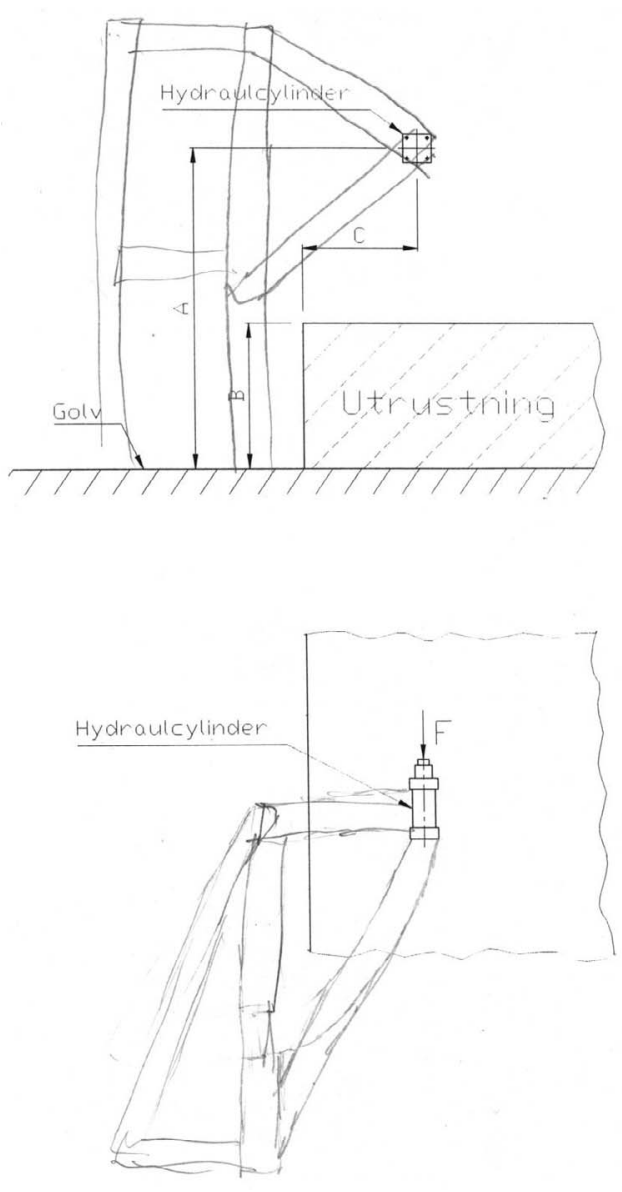

Figure 9. First sketch of a student.

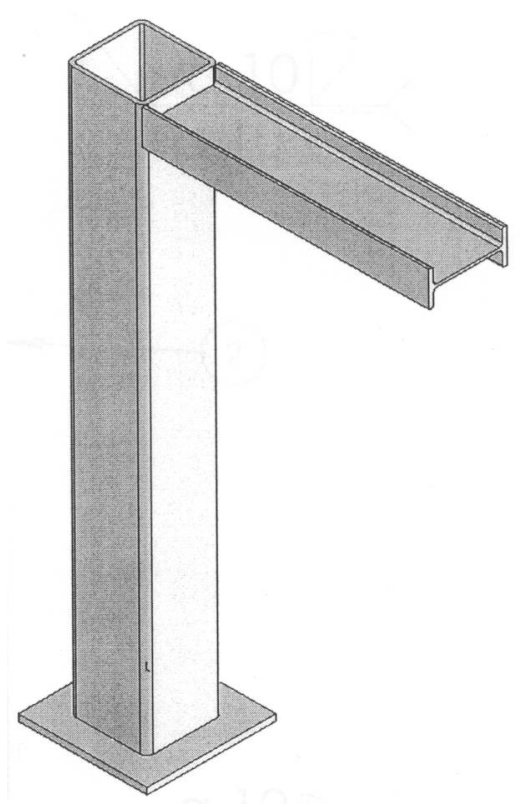

Figure 10. Final solution delivered by the same student. 
only explorative, due to the small number of designers studied, but the benefits and shortcomings of teaching by "learning by doing" and design cases study could be highlighted.

These teaching methods tend to suppress the $a d$ hocism noticed by the junior. The seniors focus more on the vital parts of the solution; they apply guidelines, and the problem-solving activity is not as loose as that of the junior observed. The interplay between synthesis and mechanical modeling seems already acquired by the student prior to the course.

Nevertheless, teaching a list of rules, principles, and guidelines with a too-general design process is not effective. The seniors neglected several points that are taken into account by the experts: problems of interface, early concretizations, and systematic choice of standard components. Moreover, both students and experts did not question the problem sufficiently, and developed very few alternatives, although they had knowledge and experience of conceptual design methods. Both took into account only a few factors (production/assembly, costs...). There is a need to develop and teach a more stringent prescriptive design process to guide the designer through the embodiment design and detail design phases, at least during the early embodiment design phase.

To this major point, other findings have been extracted from the experiments that must be taken into account for teaching the later phases of the design process. If the students rapidly assimilate the simplicity rule, the rules of safety and clarity need more time. This must be emphasized during the teaching of these rules. The students observed had a tendency not to document their work, which led to a loss of time. This can be seen as a personal organization problem, but a coupling to the vagueness inherent in design assignments cannot be excluded. The factors listed by Pahl \& Beitz [3] were insufficiently taken into account.

Finally, several points need to be further investigated, mainly the assimilation and re-use of principles, and the carrying-out of evaluations of the solutions by the students.

\section{References}

[1] C. J. Atman, J. R. Chimka, K. M. Bursic, and H. L. Nachtmann, "A comparison of freshman and senior engineering design processes”. Design Studies 20(2), 1999, pp. 131-152.

[2] R. S. Adams, J. Turns, and C. J. Atman, "Educating Effective Engineering Designers: The Role of Reflective Practice", Design Studies, vol. 24(3), 2003, pp. 275-294. [3] G. Pahl, and W. Beitz, Engineering Design - A systematic approach ( $2^{\text {nd }}$ Rev. Ed.), Springer, London, 1996. [4] D. Motte, P.-E. Andersson, and R. Bjärnemo, "A Descriptive Model of the Designer's Problem Solving Activity
During the Later Phases of the Design Process", $1^{\text {st }} C D E N$ Design Conference, 2004 (submitted).

[5] D. Motte, P.-E. Andersson, and R. Bjärnemo, "A Study of the Mechanical Designer's Strategies and Tactics During the Later Phases of the Engineering Design Process", Proceedings of the DTM/ASME 2004, Salt Lake City, 2004 (accepted).

[6] R. S. Adams, and J. Turns, "What Could Design Learning Look Like?", $6^{\text {th }}$ Design Thinking Research Symposium, Sydney, 2003.

[7] R. Oxman, "Educating the Designerly Thinker", design Studies, vol. 20(2), pp. 105-122.

[8] M. Kavakli, J. S. Gero, "Strategic Knowledge Differences Between an Expert and a Novice Designer", in U. Lindemann (Ed.), Human Behaviour in Design, Springer, pp. 42-51.

[9] J. R. Chimka, C. J. Atman, and K. M. Bursic, "Describing Student Behavior", ASEE Annual Conference Proceedings, Washington, 1996.

[10] R. S. Adams, and C. S. Atman, "Characterizing Engineering Student Design Process: An Illustration of Iteration", ASEE Annual Conference Proceedings, Charlotte, 1999.

[11] R. S. Adams, and C. S. Atman, "Cognitive Processes in Iterative Design Behavior", ASEE/IEEE Frontiers in Education Conference Proceedings, San Juan, Puerto Rico, 1999.

[12] M. C. Yang, "Concept Generation and Sketching: Correlations with Design Outcome", Proceedings of DETC/DTM, Chicago, 2003.

[13] H. Casakin \& G. Goldschmidt, "Expertise and the Use of Visual Analogy: Implications for Design Education", Design Studies, vol. 20(2), p. 153-175.

[14] D. Motte, and R. Bjärnemo, "The Cognitive Aspects of the Engineering Design Activity - A Literature Survey", Proceedings of the TMCE 2004, Lausanne, 2004.

[15] V. Hubka, and W. E. Eder, Design Science, Springer, London, 1996.

[16] D. G. Ullman, The Mechanical Design Process $\left(2^{\text {nd }}\right.$ Ed.), McGraw-Hill, New-York, 1997.

[17] S. Pugh, Total Design - Integrated Methods for Successful Product Engineering, Addison-Wesley, Reading, MA, 1990.

[18] F. G. Olsson, Produktförnyelse (Product Renewal), in Swedish, Machine Design, Lund University, Lund, 1990 (reed.)

[19] K. T. Ulrich, , and S. D. Eppinger, Product Design and Development ( $3^{\text {rd }}$ Ed.), McGraw-Hill, London, 2003.

[20] J. Sundström, R. Bjärnemo, and P.-E. Andersson, Konstruktiv utformning. Del 1: Syntes (Form giving in design. Part 1: Synthesis), in Swedish, Machine Design, Lund University, Lund, 2000.

[21] VDI, VDI 2230 Blatt 1: Systematic Calculation of High Duty Bolted Joints - Joints with One Cylindrical Bolt, VDIHandbuch Konstruktion, 2003.

[22] VDI, VDI 2225 Blatt 1: Design engineering methodics Engineering design at optimum cost - Simplified calculation of costs, VDI-Handbuch Konstruktion, 1997.

[23] B. Bergman, B. Klefsjö, Quality - from Customer Needs to Customer Satisfaction, Studentlitteratur, Lund, 2003. 
[24] E. B. Haugen, Probabilistic Mechanical Design, John Wiley \& Sons, New York, 1980.

[25] K. C. Kapur, L. R. Lamberson, Reliability in Engineering Design, John Wiley \& Sons, New York, 1977.

[26] R. Matousek, Engineering Design - A Systematic approach, Black \& Son, Glasgow, 1963. English translation by A. H. Burton, of Konstruktionslehre des allgemeinen Maschinenbaues, Springer, Berlin, 1957.

[27] A. Leyer, Maschinenkonstruktionslehre. Häfte 2: Allgemeine Gestaltungslehre, Technica-Reihe Nr. 2,

Birkhäuser, Basel/Stuttgart, 1964.

[28] M. J. French, Conceptual design for engineers ( $3^{\text {rd }}$ Ed.), Springer, London, 1998.

[29] D. Motte, P.-E. Andersson, and R. Bjärnemo, "Study of the Designer's Cognitive Processes During the Later Phases of the Engineering Design Process", Proceedings of the Design 2004, Dubrovnik, 2004, pp. 421-428.

[30] K. A. Ericsson, and H. A. Simon, Protocol Analysis: Verbal Report as Data (Rev. Ed.), MIT Press, Cambridge, MA, 1993.

[31] C. J. Atman, K. M. Bursic, and S. L. Lozito, “An Application of Protocol Analysis to the Engineering Design Process", ASEE Annual Conference Proceedings, 1996.

[32] B. Bender, L. Blessing, "On the Superiority of Opportunistic Design Strategies during Early Embodiment Design”, Proceedings of the Design 2004, Dubrovnik, 2004, pp. 117-122. 